\title{
Calibration-measurement unit for the automation of vector network analyzer measurements
}

\author{
I. Rolfes ${ }^{1}$, B. Will ${ }^{2}$, and B. Schiek ${ }^{2}$ \\ ${ }^{1}$ Institut für Hochfrequenztechnik und Funksysteme, Leibniz Universität Hannover, Appelstraße 9A, 30167 Hannover, \\ Germany \\ ${ }^{2}$ Institut für Hochfrequenztechnik, Ruhr-Universität Bochum, Universitätsstraße 150, 44801 Bochum, Germany
}

\begin{abstract}
With the availability of multi-port vector network analyzers, the need for automated, calibrated measurement facilities increases. In this contribution, a calibrationmeasurement unit is presented which realizes a repeatable automated calibration of the measurement setup as well as a user-friendly measurement of the device under test (DUT). In difference to commercially available calibration units, which are connected to the ports of the vector network analyzer preceding a measurement and which are then removed so that the DUT can be connected, the presented calibrationmeasurement unit is permanently connected to the ports of the VNA for the calibration as well as for the measurement of the DUT. This helps to simplify the calibrated measurement of complex scattering parameters. Moreover, a full integration of the calibration unit into the analyzer setup becomes possible. The calibration-measurement unit is based on a multiport switch setup of e.g. electromechanical relays. Under the assumption of symmetry of a switch, on the one hand the unit realizes the connection of calibration standards like one-port reflection standards and two-port through connections between different ports and on the other hand it enables the connection of the DUT. The calibration-measurement unit is applicable for two-port VNAs as well as for multiport VNAs. For the calibration of the unit, methods with completely known calibration standards like SOLT (short, open, load, through) as well as self-calibration procedures like TMR or TLR can be applied.
\end{abstract}

\section{Introduction}

For the automation of the calibration procedure of vector network analyzers, complex calibration units based on calibration methods with completely known calibration standards, like SOLT (Short, Open, Load, Thru) are already commer-

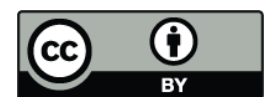

Correspondence to: I. Rolfes (rolfes@hft.uni-hannover.de)

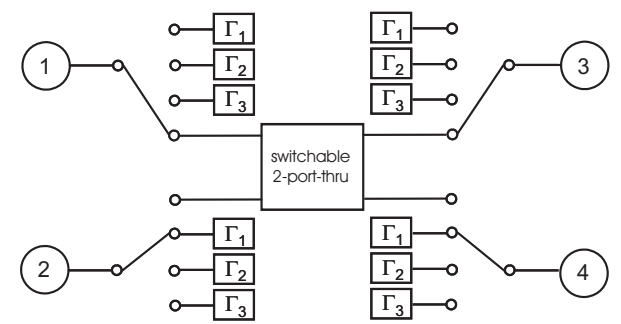

Fig. 1. Example of a calibration unit for a 4-port-analyzer.

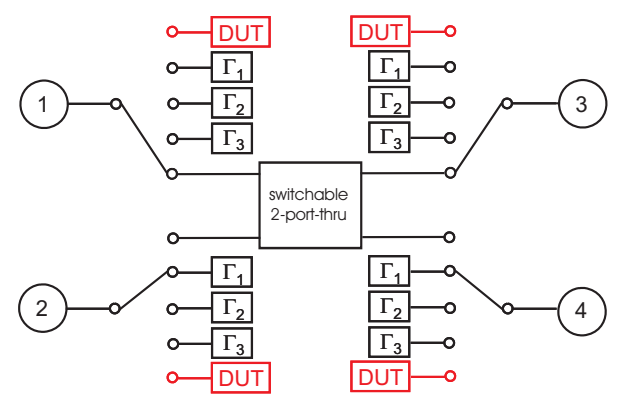

Fig. 2. Setup of the calibration-measurement unit.

cially available (Henkel, 2006; Krekels and Schiek, 1995; Krekels, 1996). In Fig. 1 a possible setup of a calibration unit for a 4-port-analyzer is shown. As can be deduced from the number of necessary calibration standards in Fig. 1, an automation of the calibration procedure is desirable and also recommendable in order to avoid calibration errors caused by e.g. connecting errors. The various standards are connected consecutively to the ports of the analyzer by a switch. For this purpose, the calibration standards have to be known exactly. After calibration the device under test (DUT) can be connected to the ports of the analyzer, and the scattering parameters of the DUT can be determined error-corrected in relation to the chosen phase reference plane, e.g. in the phase plane of the DUT's connectors. 


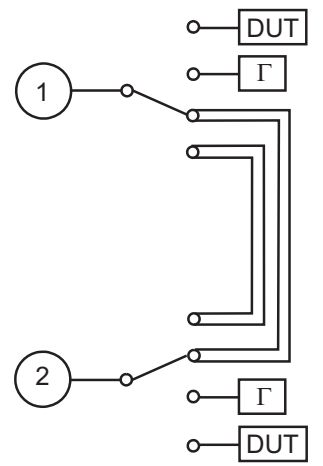

Fig. 3. Combined calibration measurement setup based on a TLRcalibration.

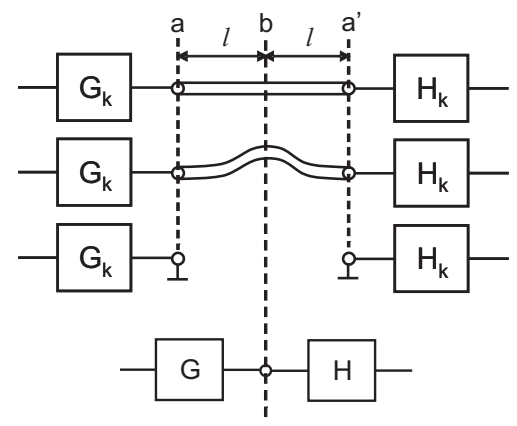

Fig. 4. Two-port error model of the VNA with TLR-calibration standards.

\section{Calibration-measurement unit}

For the automation of both the calibration of the analyzer and the measurement of the scattering parameters of a DUT, a combined setup, a so-called calibration-measurement unit is introduced. A setup for 4-port-measurements is shown in Fig. 2. The ports 1 to 4 are connected to the vector network analyzer and the DUT is connected to the ports named DUT. Thus, a calibration-measurement unit results, where the calibration standards do not have to be removed by the user in order to connect the DUT to the analyzer. The user can directly connect the DUT to the resulting measurement ports, thus leading to a user-friendly setup, which can easily be recalibrated without having to replace the DUT. However, as a precondition for this combined setup, the different switch positions of a switch have to be symmetrical, so that the phase reference planes of the measured scattering parameters lie behind the switches in the phase plane of the DUT. As a consequence, the non-idealities of the switch are included in the error terms representing the systematic errors of the network analyzer. The calibration can either be performed based on calibration methods with completely known calibration standards as e.g. SOLT as shown in Fig. 2 or with partly unknown calibration standards like TLR (Engen and Hoer, 1979; Eul and Schiek, 1991) as shown in Fig. 3 for a two-port setup. As the phase reference planes are preferably chosen in the plane



Fig. 5. Extension of setup for multiport analyzers.

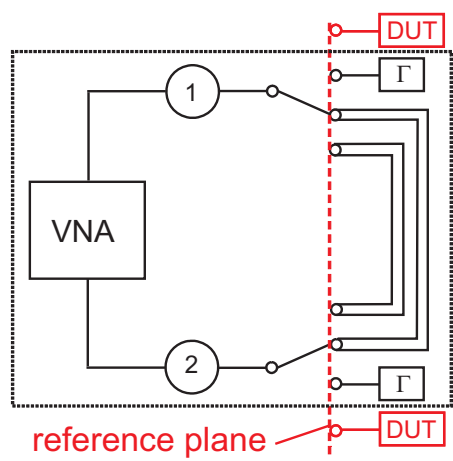

Fig. 6. Network analyzer with integrated calibration unit and measurement port for the contacting of the DUT.

of the DUT, for the TLR calibration a transformation of the phase reference plane is necessary.

This transformation can most easily be realized by choosing the reflection standard $\Gamma$ for the TLR calibration as a short or open standard in the desired reference planes $a, a^{\prime}$ as illustrated in Fig. 4.

The error two-ports $G$ and $H$ in Fig. 4 are known after a TLR calibration. The error two-ports $G_{k}$ and $H_{k}$ for the transformed reference planes $a, a^{\prime}$ are calculable by

$\left[G_{k}\right]=[G]\left[\begin{array}{cc}e^{-\gamma l} & 0 \\ 0 & e^{\gamma l}\end{array}\right]$

with

$e^{2 \gamma l}=\frac{\rho}{\Gamma}$

where $\rho$ is the reflection coefficient of the TLR reflection standard in the reference plane $b$, which is known from selfcalibration after the TLR calibration, and $\Gamma$ is the reflection coefficient in reference planes $a$ and $a^{\prime}$, which here is chosen as $\Gamma=-1$ for the termination with a short. Based on Eq. (1) for $G_{k}$ and a similar equation for $H_{k}$ the phase reference plane for the measurements can thus be moved into the phase plane of the DUT. The measured scattering parameters refer directly to the DUT without need for a deembedding. 


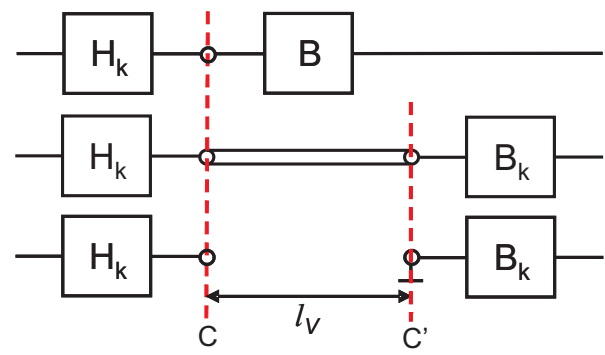

Fig. 7. Two-port setup for elimination of influence of additional cables.



Fig. 8. Photo of the calibration measurement unit. $\%$ vspace- $3 \mathrm{~mm}$

The TLR-calibration setup of Fig. 4 as well as the previously presented calibration setups based on SOLT can easily be adopted to multiport analyzer systems by adding further line connections to the calibration measurement unit as shown in Fig. 5. In case that these lines are of unknown length but of identical geometry and line impedance as the previously used line standards for TLR calibration, it is possible to determine this unknown line length from an additional reflection measurement.

\section{Integration into an analyzer system}

The calibration standards can be integrated completely into a network analyzer system, so that only the measurement port for the contacting of the DUT remains as an interface to the user. This is demonstrated in Fig. 6. This setup allows an automated calibration of the measurement system without being in need for a manuel interaction of the user. On the one hand, this helps to reduce the required knowledge about calibration technology of the user and on the other hand, this possibility of automated calibration and combined measurement can furthermore be useful for applications where no user interaction is possible in the measurement plane, as e.g. in industrial drill hole applications where the measurement plane can be deep under earth's surface.

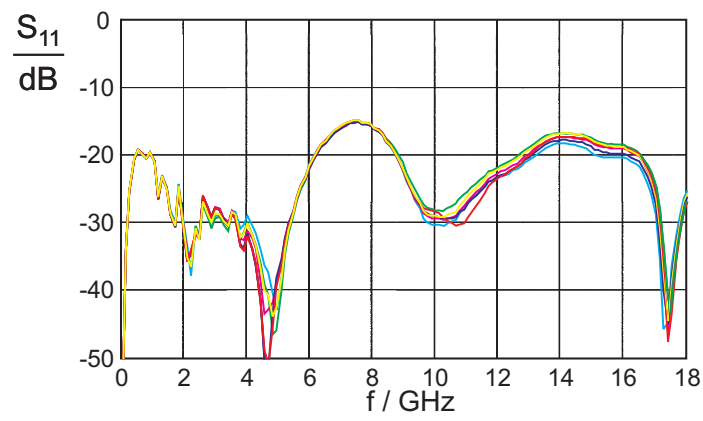

Fig. 9. Verification of the symmetry of the switch. Comparison of $S_{11}$ measurements for all 6 positions of the switch.

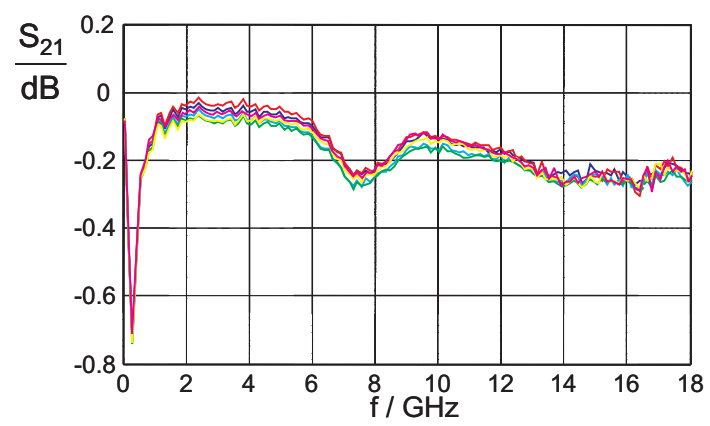

Fig. 10. Verification of the symmetry of the switch. Comparison of $S_{21}$ measurements for all 6 positions of the switch.

For the case that additional cables are necessary for the contacting of the DUT, it is possible to eliminate the influence of the cables by performing two additional measurements: first, the measurement of the transmission of the cables and secondly, the measurement of the cables with a reflection standard placed in the phase reference plane. The setup is shown exemplarily in Fig. 7. Similarly to the previously described multiport extension, the influence of the cables can be corrected.

\section{Measurement results}

In order to verify the functionality of the proposed calibration-measurement unit, a demonstrator was realized on the basis of electromechanical relays as shown in Fig. 8. For verification of the symmetry of a switch, the different positions of a switch are compared. The measurements of the scattering parameters $S_{11}$ and $S_{21}$ in Figs. 9 and 10 show a good agreement of the high-frequency behavior for the different switching positions.

In Figs. 11 and 12, some further results for the verification of the switches symmetry are given. In this case, the scattering parameters are measured for varying terminations like open, short, match and the repetition of contacting.

In Figs. 13 and 14, the calibrated measurements of the scattering parameters $S_{11}$ and $S_{21}$ of a $3 \mathrm{~dB}$-attenuator are 


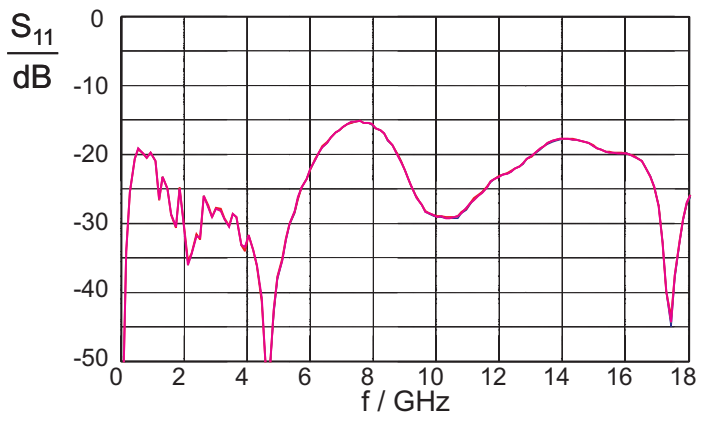

Fig. 11. Verification of the symmetry of the switch. Comparison of measurements of $S_{11}$ while contacting varying terminations like open, match and repetition of contacting.

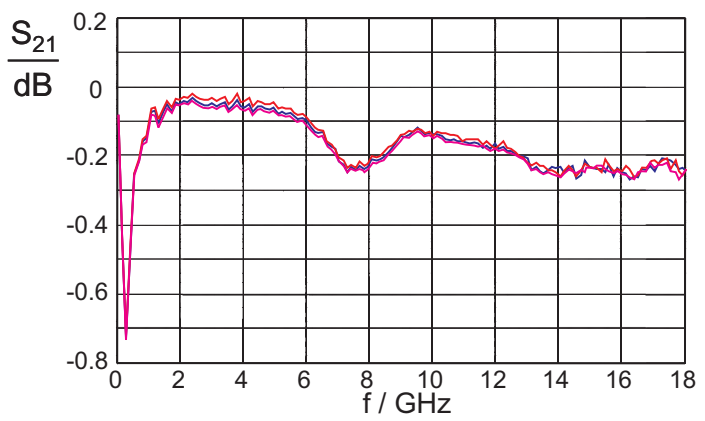

Fig. 12. Verification of the symmetry of the switch. Comparison of measurements of $S_{21}$ while contacting varying terminations like open, match, and repetition of contacting.

shown exemplarily. A comparison of a direct measurement of the DUT and the measurement with the help of the calibration-measurement unit proves the robust functionality of the developed method.

\section{Summary}

A calibration-measurement unit is presented which enables on the one hand automated, repeatable calibrations of vector network analyzers and on the other hand the measurement of scattering parameters of DUTs. Well-known calibration methods like SOLT as well as self-calibration methods like TLR are applicable. The possibility of integration of the whole setup is described, leading to a measurement system where the user can directly connect the DUT to the measurement ports of the system, while the calibration standards can be left in the system so that a repetition of calibration is possible at every time. The setup is based on switching multiports, which have to be symmetrical individually for the proposed setup. For the verification of the robust functionality of the calibration-measurement unit a demonstrator and measurement results are presented.

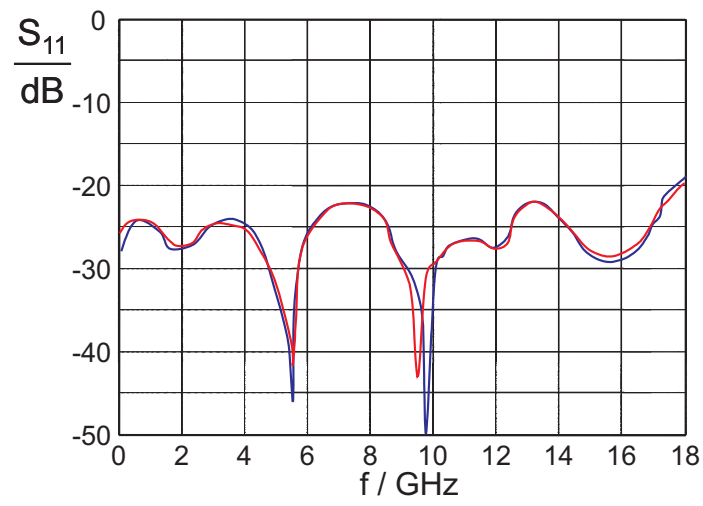

Fig. 13. Comparison of the measurement of $S_{11}$ of a $3 \mathrm{~dB}$-attenuator based on a direct TLR-calibration (blue line) and based on the calibration-measurement-setup (red line).

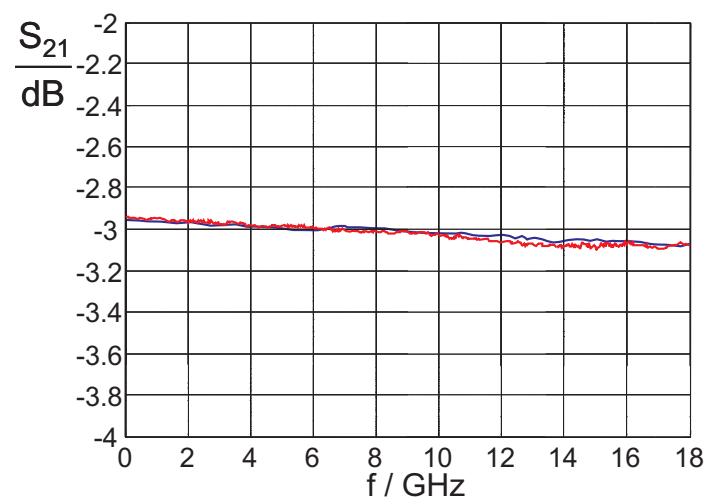

Fig. 14. Comparison of the measurement of $S_{21}$ of a $3 \mathrm{~dB}$-attenuator based on a direct TLR-calibration (blue line) and based on the calibration-measurement-setup (red line).

\section{References}

Engen, G. F. and Hoer, C. A.: Thru-Reflect-Line: An improved technique for calibrating the dual six port automatic network analyzer, IEEE T. Microw. Theory, 27, 987-993, 1979.

Eul, H. J. and Schiek, B.: A Generalized Theory and New Calibration Procedures for Network Analyzer Self-Calibration, IEEE T. Microw. Theory, 39, 724-731, 1991.

Henkel, A.: Unrivaled - up to eight test ports in a single unit, News from Rohde \& Schwarz, 189, 26-29, 2006.

Krekels, H.-G.: AutoKal: Automatic Calibration of Vector Network Analyzer ZVR, Rohde \& Schwarz Application Note 1EZ30 IE, 1996.

Krekels, H.-G. and Schiek, B.: A novel Procedure for an automatic Network-Analyzer Calibration, IEEE T. Instrum. Meas., 44, 291-294, 1995. 